\title{
Ciclagem de nutrientes em povoamento de Eucalyptus dunnii Maiden: produção de serapilheira e devolução de macronutrientes no bioma Pampa ${ }^{1}$
}

\author{
Claudinei Garlet ${ }^{2}$; Mauro Valdir Schumacher ${ }^{3}$; Grasiele Dick ${ }^{4}$; Márcio Viera ${ }^{5}$
}

Resumo: A serapilheira representa um componente de extrema importância na dinâmica nutricional dos ecossistemas florestais, atuando como um agente regulador dos processos de entradas e saídas dos elementos minerais e orgânicos na interface solo-planta. Diante disso, o presente trabalho objetivou caracterizar a produção da serapilheira e a devolução de macronutrientes em um povoamento de Eucalyptus dunnii com cinco anos de idade, na região da campanha sudoeste do bioma Pampa, município de Alegrete, Rio Grande do Sul, Brasil. Para isso, foram instaladas quatro parcelas de $20 \mathrm{~m}$ x 21 $\mathrm{m}$, distribuindo-se, em cada uma delas, quatro coletores de área com $0,5 \mathrm{~m}^{2}$. Além disso, dentro de cada parcela foram demarcadas quatro áreas de 7,0 $\mathrm{m}^{2}$ para coleta de galhos grossos (diâmetro $>0,51 \mathrm{~cm}$ ). As amostras de serapilheira foram coletadas quinzenalmente e separadas nas frações folhas, miscelânea, galhos finos (diâmetro $<0,5 \mathrm{~cm}$ ) e galhos grossos, sendo posteriormente secas em estufa, pesadas, moídas e submetidas à análise química. A produção total de serapilheira foi de $9,6 \mathrm{Mg} \mathrm{ha}^{-1}$, onde a fração folhas é a maior constituinte $(52,7 \%$ do total). As quantidades de $\mathrm{Ca}, \mathrm{N}, \mathrm{K}, \mathrm{Mg}, \mathrm{S}$ e $\mathrm{P}$ na serapilheira foram de 109,$36 ; 59,62 ; 33,99 ; 21,17 ; 5,41$ e $3,93 \mathrm{~kg} \mathrm{ha}^{-1}$, respectivamente, dos quais $72,4 \%$ do total foi representado pelo somatório de $\mathrm{Ca}+\mathrm{N}$. Portanto, a serapilheira desse povoamento atua na manutenção da fertilidade do solo, o que é importante, já que não se trata de um ambiente natural.

Palavras - chave: Povoamento comercial; Silvicultura; Matéria orgânica; Nutrição florestal

\section{Nutrient cycling in Eucalyptus dunnii Maiden: litterfall and macronutrient return in Pampa biome}

\begin{abstract}
The litterfall represents a compartment of extreme importance in the nutritional dynamics of forest ecosystems, acting as an agent regulating the processes of inputs and outputs of the mineral and organic elements at the soil-plant interface. The objective of this work was to characterize litterfall and the return of macronutrients to a five - year - old Eucalyptus dunnii stand in Pampa biome region of Alegrete city, Rio Grande do Sul state, Brazil. For this, four plots of 20 $\mathrm{m} \times 21 \mathrm{~m}$ were installed, each of which had four area collectors with $0.5 \mathrm{~m}^{2}$. In addition, within each plot four areas of 7.0 $\mathrm{m}^{2}$ were collected for collection of branches (diameter $>0.51 \mathrm{~cm}$ ). The litterfall samples were collected biweekly and separated into leaf, miscellaneous, twigs (diameter $<0.5 \mathrm{~cm}$ ) and branches fractions, then dried in greenhouses, weighed, milled and subjected to chemical analysis. The total litterfall was $9.6 \mathrm{Mg} \mathrm{ha}^{-1}$, in which the leaf fraction was the largest constituent (52.7\% of the total). The amounts of $\mathrm{Ca}, \mathrm{N}, \mathrm{K}, \mathrm{Mg}, \mathrm{S}$ and $\mathrm{P}$ in litterfall were $109.36 ; 59.62 ; 33.99 ; 21.17 ; 5.41$ and $3.93 \mathrm{~kg} \mathrm{ha}^{-1,}$ respectively, of which $72.4 \%$ of the total was represented by the sum of $\mathrm{Ca}+\mathrm{N}$. Therefore, the litterfall of this stand acts in the maintenance of soil fertility, which is important, since it is not a natural environment.
\end{abstract}

Keywords: Commercial stand; Silviculture; Organic matter; Forest nutrition

\footnotetext{
${ }^{1}$ Recebido em 25.02.2019 e aceito para publicação como artigo científico em 26.03.2019.

${ }^{2}$ Engenheiro Florestal. Mestrando no Programa de Pós-Graduação em Engenharia Florestal, Universidade Federal de Santa Maria, Rio Grande do Sul, RS. E-mail: <claudineigarlet@gmail.com>

${ }^{3}$ Engenheiro Florestal, Dr.nat.techn. Professor Titular do Departamento de Ciências Florestais, Universidade Federal de Santa Maria, Rio Grande do Sul, RS. E-mail: <mauro.schumacher@ufsm.br>

${ }^{4}$ Engenheira Florestal, Dra. Pós-doutoranda no Programa de Pós-Graduação em Engenharia Florestal, Universidade Federal de Santa Maria, Rio Grande do Sul, RS. E-mail: <grasidick@ hotmail.com>

${ }^{5}$ Engenheiro Florestal, Dr. Professor Adjunto do Colégio Politécnico, Universidade Federal de Santa Maria, Rio Grande do Sul, RS. Email: <marcio.viera@ufsm.br>
} 


\section{Introdução}

A introdução das plantações com espécies arbóreas de rápido crescimento, principalmente dos gêneros Pinus e Eucalyptus, se deu basicamente no início dos anos 60, no período de vigência dos incentivos fiscais. A adoção desse programa teve por objetivo suprir a indústria de celulose e papel com madeira de plantações florestais e, posteriormente, para outros segmentos, como a produção de painéis, siderurgia e secagem de grãos (MOREIRA et al., 2017).

Considerando que a demanda por madeira tende a alcançar valores superiores aos atuais, se torna evidente a necessidade de ampliação das áreas reflorestadas, visando o atendimento à essas demandas. No entanto, em meio a esse processo de progressão dos plantios florestais, muitas das áreas destinadas à silvicultura com eucalipto no bioma Pampa estão localizadas em regiões com solos sujeitos à arenização e de baixa fertilidade, que, até então, estavam sendo utilizadas pela pecuária, um tipo de atividade que torna os solos ainda mais suscetíveis à erosão (MARCHIORI, 2002).

Nessas áreas, estudos sobre manejo de solo e adubação, ciclagem de nutrientes e outros processos que alteram o desenvolvimento da espécie cultivada, se tornam necessários. Dentre esses, a ciclagem de nutrientes apresenta relevância, porque se caracteriza como o mais importante processo de transferência de nutrientes para o solo, o qual possibilita, dessa forma, o crescimento de árvores e o desenvolvimento de florestas em solos de baixa fertilidade natural (VIERA e SCHUMACHER, 2010b).

Por meio de estudos acerca da ciclagem de nutrientes em ecossistemas florestais, são obtidas informações que irão auxiliar no entendimento da dinâmica nutricional que ocorre nesses ambientes, permitindo criar novas técnicas de manejo florestal para serem utilizadas em áreas de recuperação, bem como em áreas que visam à produção de madeira, mantendo a produtividade desses sítios. Diante disso, o objetivo do presente estudo foi caracterizar a produção de serapilheira e a devolução de nutrientes em um povoamento de Eucalyptus dunnii estabelecido na região da campanha sudoeste do bioma Pampa, Rio Grande do Sul, Brasil.

\section{Material e métodos}

Caracterização do sítio

O presente trabalho foi realizado durante o ano de 2014, em uma área cultivada com Eucalyptus dunnii na região da campanha sudoeste do bioma Pampa, município de Alegrete, Rio Grande do Sul, Brasil (Figura 1). Segundo a classificação climática de Köppen, o clima da região é do tipo $\mathrm{Cfa}$, com temperatura média anual de $18,6^{\circ} \mathrm{C}$ e precipitação média anual de $1574 \mathrm{~mm}$ (ALVARES et al., 2014).

Conforme o Sistema Brasileiro de Classificação de Solos (EMBRAPA, 2013), na área de estudo o solo é do tipo Argissolo Vermelho Distrófico típico. Na Tabela 1 estão apresentadas algumas propriedades químicas do solo, dentre as quais se destaca a baixa fertilidade, uma vez que há baixos teores de matéria orgânica, $\mathrm{Ca}$ e $\mathrm{Mg}$; $\mathrm{pH}$ muito baixo; saturação por bases (V) muito baixa e saturação por alumínio (m) alta (CQFS - RS/SC, 2016). Quanto às características do solo, o mesmo apresenta textura arenosa variando de francoarenosa, com média de $77 \%$ de areia na profundidade de $0-40 \mathrm{~cm}$, passando para franco-argilo-arenosa, com média de $69 \%$ de areia nas camadas na profundidade entre 40$100 \mathrm{~cm}$ (SILVA, 2014).

Para a implantação do povoamento, foi realizado o preparo do solo, com uma subsolagem a aproximadamente $50 \mathrm{~cm}$ de profundidade. A aplicação de fertilizante ocorreu em três fases: no plantio $\left(300 \mathrm{~kg} \mathrm{ha}^{-1}\right.$ do formulado $06-30-60)$, 90 dias após o plantio (140 kg ha ${ }^{-1}$ do formulado $22-05-$ $20)$, e aos 270 dias após o plantio (140 kg ha ${ }^{-1}$ do formulado $22-00-18)$. 


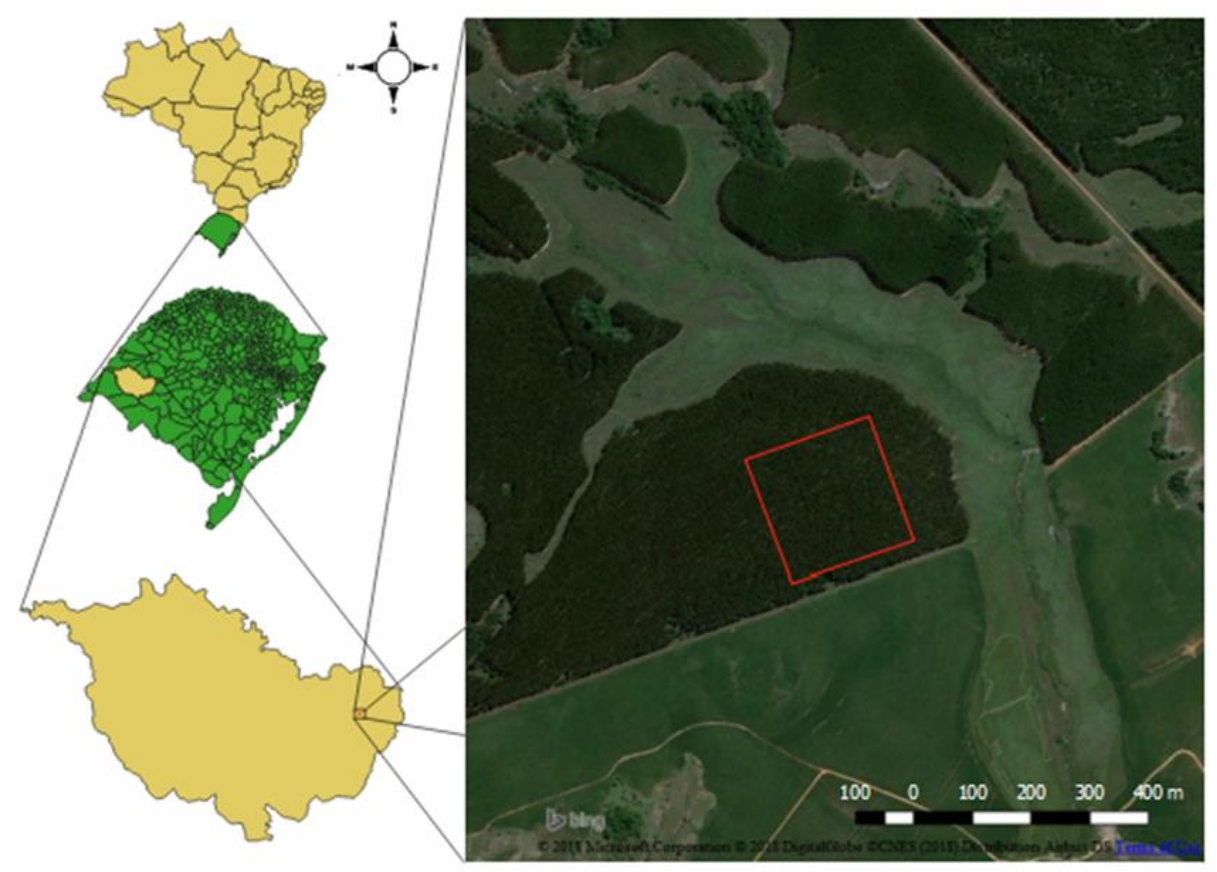

Figura 1 - Localização da área de estudo no município de Alegrete - RS.

Figure 1 - Location of the study area in the city of Alegrete - RS state.

Tabela 1 - Características químicas do solo em povoamento de Eucalyptus dunnii.

Table 1 - Chemical soil characteristics of Eucalyptus dunnii stand.

\begin{tabular}{|c|c|c|c|c|c|c|c|c|c|}
\hline \multirow{2}{*}{$\begin{array}{l}\text { Prof. } \\
(\mathrm{cm})\end{array}$} & \multirow{2}{*}{$\underset{\text { (água) }}{\mathrm{pH}}$} & \multirow{2}{*}{$\begin{array}{c}\operatorname{MOS}^{*} \\
(\%)\end{array}$} & $\mathrm{P}$ & $\mathrm{K}$ & $\mathrm{Ca}$ & $\mathrm{Mg}$ & $\mathrm{Al}$ & $\mathrm{m}$ & V \\
\hline & & & \multicolumn{2}{|c|}{$\mathrm{mg} \mathrm{dm^{-3 }}$} & \multicolumn{3}{|c|}{$\mathrm{cmol}_{\mathrm{c}} \mathrm{dm}^{-3}$} & \multicolumn{2}{|c|}{$\%$} \\
\hline $0-20$ & 4,74 & 1,00 & 1,8 & 20 & 0,4 & 0,3 & 1,9 & 71 & 9,07 \\
\hline $20-40$ & 4,84 & 0,91 & 1,6 & 12 & 0,7 & 0,2 & 1,9 & 65 & 10,9 \\
\hline $40-60$ & 4,96 & 0,85 & 1,4 & 9,6 & 1,2 & 0,2 & 1,9 & 55 & 15,1 \\
\hline $60-80$ & 4,98 & 0,78 & 1,4 & 8,3 & 1,8 & 0,3 & 2,0 & 48 & 20,5 \\
\hline $80-100$ & 5,00 & 0,72 & 1,5 & 8,3 & 1,8 & 0,4 & 1,7 & 43 & 21,2 \\
\hline
\end{tabular}

*MOS = matéria orgânica do solo; $m$ = Saturação por alumínio; V = Saturação por bases. Fonte: Silva (2014).

Amostragem da serapilheira

Para a quantificação da serapilheira produzida, foram demarcadas quatro parcelas com dimensões de $20 \mathrm{~m}$ x $21 \mathrm{~m}$. Em cada parcela, instalaram-se quatro coletores com área útil de $0,5 \mathrm{~m}^{2}$. Os coletores foram dispostos em quatro posições diferentes dentro de cada parcela: linha, entrelinha, diagonal e encostado ao tronco de uma árvore.

Durante o ano de 2014, quando o povoamento estava com 60 meses de idade, a serapilheira depositada nos coletores foi recolhida quinzenalmente, sendo esta, posteriormente, encaminhada para separação das frações folhas, miscelânea (casca e material reprodutivo) e galhos finos (diâmetro menor que $0,5 \mathrm{~cm}$ ).

Para a coleta de galhos grossos (diâmetro maior que $0,51 \mathrm{~cm}$ ), delimitaram-se, dentro de cada parcela, quatro locais de coleta com área útil de $7,0 \mathrm{~m}^{2}$ cada. As amostras quinzenais 
foram reunidas, para formação de amostras compostas mensais, que passaram por processo de secagem em estufa de circulação forçada de ar, a $70^{\circ} \mathrm{C}$ até peso constante, com posterior pesagem em balança de precisão $(0,01 \mathrm{~g})$ para determinação da massa seca.

Análises químicas

As amostras secas foram moídas em moinho tipo Willey, para determinação dos teores dos seguintes macronutrientes: $\mathrm{N}, \mathrm{P}, \mathrm{K}, \mathrm{Ca}$ e $\mathrm{Mg}$. O nitrogênio foi determinado pelo método Kjeldahl (digestão sulfúrica $=\mathrm{H}_{2} \mathrm{SO}_{4}+\mathrm{H}_{2} \mathrm{O}_{2}$ ); $\mathrm{P}$ foi determinado por espectrometria (digestão nítrica-perclórica); $\mathrm{K}$ foi determinado por fotometria de chama; e o $\mathrm{Ca}$ e $\mathrm{Mg}$ por espectrofotometria de absorção atômica (digestão nítrica-perclórica) segundo a metodologia de Miyazawa et al. (1999) e Tedesco et al. (1995).

Procedimentos estatísticos

Para a realização das análises foi considerado um delineamento inteiramente casualizado com quatro repetições, as diferentes frações formadoras da serapilheira (folhas, galhos finos, miscelânea e ramos) como tratamentos, e os meses como repetições (12 meses). Foi aplicado o teste de Tukey a 5\% de probabilidade de erro. A análise estatística foi realizada utilizando o programa Assistat 7.7 ( (SILVA e AZEVEDO, 2002).

\section{Resultados e Discussão}

Produção de serapilheira

A produção total de serapilheira foi de 9,6 $\mathrm{Mg} \mathrm{ha}{ }^{-1}$. Viera et al. (2014), estudando a dinâmica nutricional em um povoamento de Eucalyptus urophylla $x$ Eucalyptus globulus, observaram que a produção de serapilheira foi crescente com o aumento da idade do povoamento, sendo 6,9 $\mathrm{Mg} \mathrm{ha}^{-1}$ aos seis anos e 8,5 $\mathrm{Mg} \mathrm{ha}^{-1}$ aos nove anos. Inkotte et al. (2015) encontraram uma produção média anual de serapilheira de $8,5 \mathrm{Mg} \mathrm{ha}^{-1}$ em povoamento de Eucalyptus dunnii no oeste de Santa Catarina, Sul do Brasil. Enquanto que, Corrêa et al. (2013) registraram a deposição de aproximadamente 4,1 $\mathrm{Mg} \mathrm{ha}^{-1}$ em um povoamento de Eucalyptus dunnii, no qual a menor quantidade de serapilheira pode ser explicada pela menor idade do povoamento quando comparado ao presente estudo.

Com os trabalhos citados acima, percebe-se a variabilidade dos resultados na deposição de serapilheira. Isto se deve à atuação dos mais diversos fatores abióticos e bióticos, como tipo de vegetação, altitude, latitude, precipitação, temperatura, regimes de luminosidade, relevo, disponibilidade hídrica e características do solo (FIGUEIREDO FILHO et al., 2003), sendo o clima um dos fatores mais importantes. Cabe ressaltar que, o maior valor da produção de serapilheira encontrado, em comparação com os demais estudos, está relacionado à expressiva contribuição dos galhos grossos no total de material depositado.

Durante o período de monitoramento, observaram-se diferenças entre as quantidades das frações em relação ao período de avaliação (Figura 2). Fevereiro foi o mês com a maior produção de serapilheira $\left(1,55 \mathrm{Mg} \mathrm{ha}{ }^{-1}\right)$ enquanto que, as menores produções ocorreram em julho $\left(0,35 \mathrm{Mg} \mathrm{ha}^{-1}\right)$.

De maneira geral, os maiores valores de deposição foram observados nos meses de novembro, dezembro, janeiro e fevereiro. Essa variação anual na produção de serapilheira está ligada aos fatores climáticos que atuam dentro do ecossistema, principalmente a temperatura. Tal fato também foi observado por Balieiro et al. (2004), ao estudarem a deposição de serapilheira em plantios de Pseudosamanea guachapele e Eucalyptus grandis, verificaram maiores taxas de deposição nos meses com temperatura mais elevada.

A fração folhas foi a que apresentou maior deposição, representando $52,7 \%$ da serapilheira total, seguida de galhos grossos $(23,7 \%)$, galhos finos $(13,9 \%)$ e miscelânea $(9,7 \%)$. Segundo Viera e Schumacher (2010b), o aumento da 
temperatura do ar no período do verão, aliado à baixa intensidade pluviométrica poderia ocasionar elevação da deposição foliar como estratégia da sobrevivência das plantas, evitando, assim, a perda de água através da alta intensidade transpiratória causada pelo calor.

Folhas
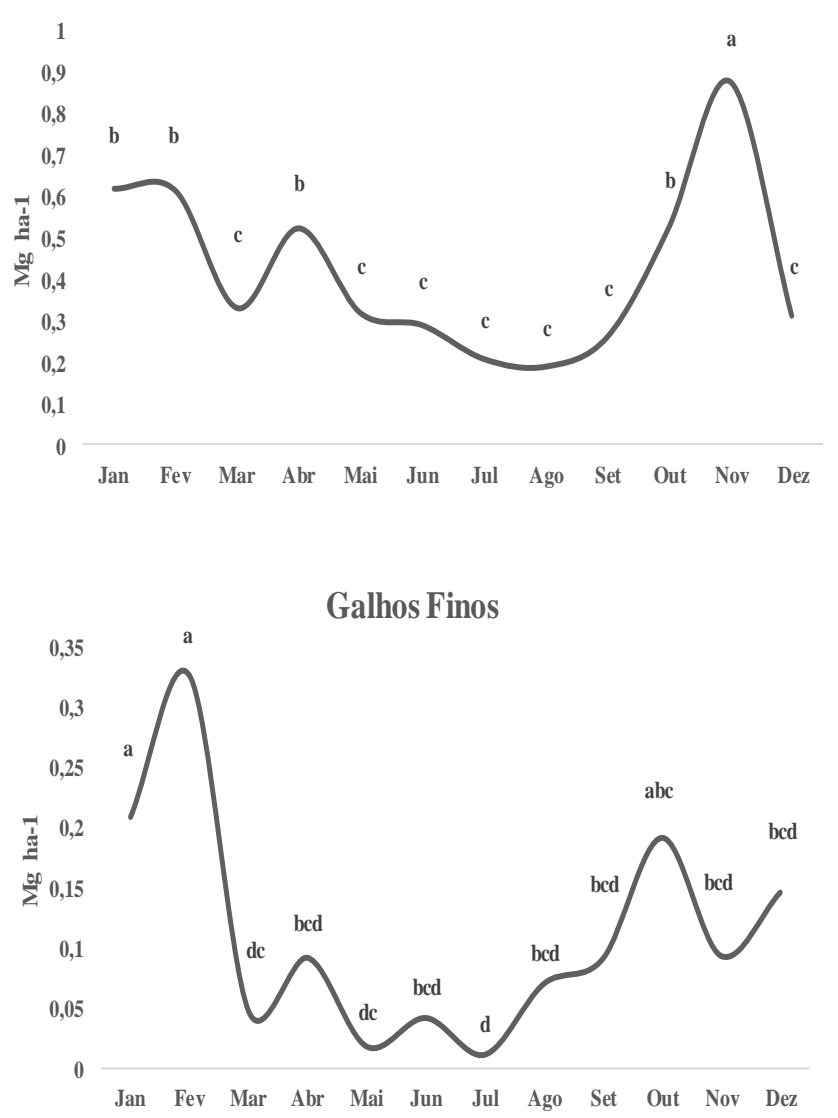

Assim como no presente estudo, Figueiredo Filho et al. (2003), Toledo e Pereira (2004), Corrêa et al. (2013), Tang et al. (2013) e Cizungu et al. (2014) encontraram valores superiores a $50 \%$ da fração folhas com relação ao total de serapilheira produzida.
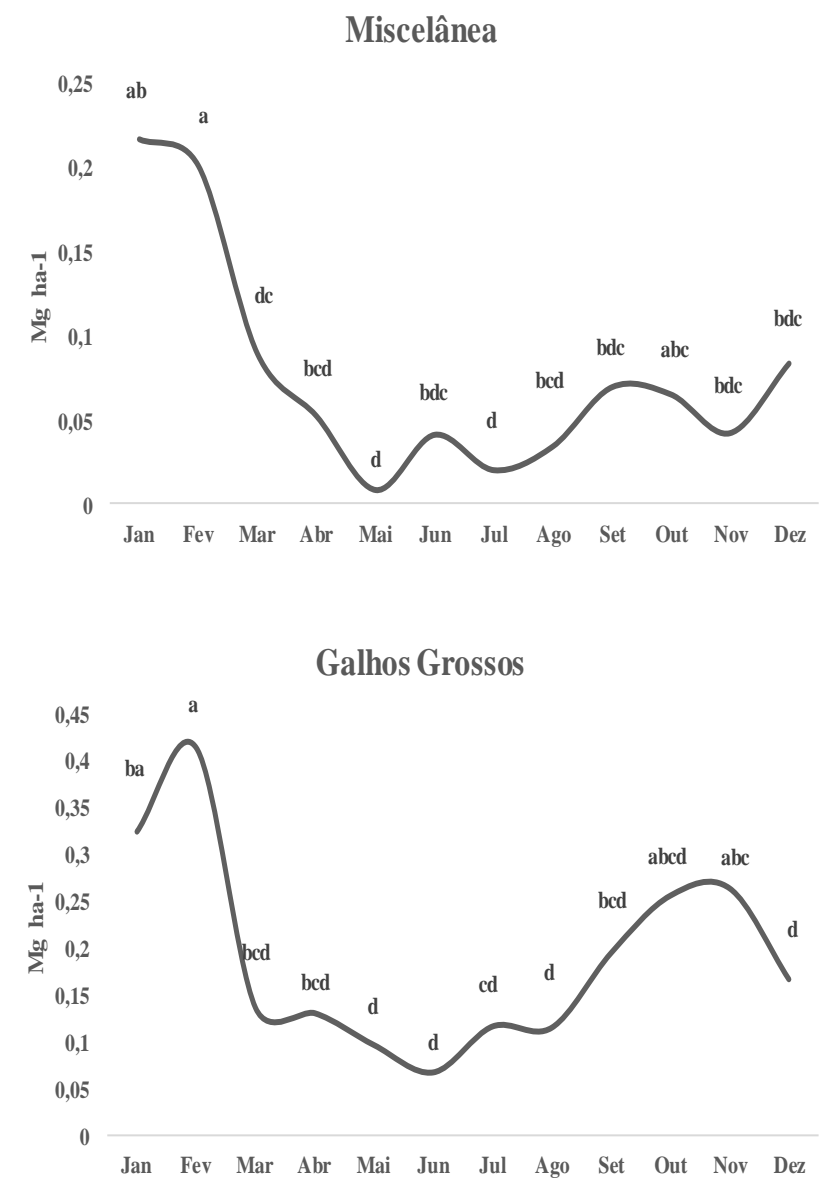

Total

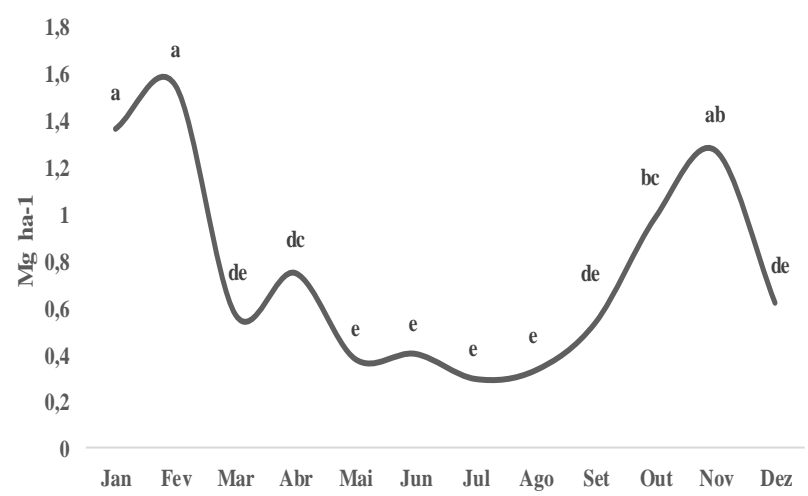

Figura 2 - Produção média mensal das frações da serapilheira produzida em povoamento de Eucalyptus dunnii, Alegrete, RS.

Figure 2 - Average monthly yield of the fractions of litterfall in Eucalyptus dunnii stand, Alegrete, RS state. 
Para a fração miscelânea, janeiro foi o mês com a maior produção, com $0,22 \mathrm{Mg} \mathrm{ha}^{-1}$, e o mês com a menor produção foi maio, com um total de $0,01 \mathrm{Mg} \mathrm{ha}^{-1}$. Para a fração galhos finos, a maior produção ocorreu no mês de fevereiro, com 0,32 $\mathrm{Mg} \mathrm{ha}^{-1}$ e a menor em julho com $0,01 \mathrm{Mg} \mathrm{ha}^{-1}$. Na fração galhos grossos, a maior produção foi em fevereiro, com $0,41 \mathrm{Mg}$ $\mathrm{ha}^{-1}$ e o mês com menor produção foi junho, com um total de 0,07 $\mathrm{Mg} \mathrm{ha}^{-1}$.

Devolução de nutrientes

\section{Concentrações de macronutrientes}

A fração folhas foi a que apresentou as maiores concentrações de nutrientes quando comparada aos demais componentes (Tabela 2). Segundo Viera e Schumacher (2010a), as folhas apresentam essa maior tendência de concentrar nutrientes devido ao fato destas apresentarem a maior atividade metabólica, além de possuírem a maioria das células que são responsáveis pela fotossíntese e transpiração.
Em estudo realizado por Carvalho (2014) em um povoamento com o híbrido de Eucalyptus urophylla $x$ Eucalyptus grandis, foi observado que mais de $50 \%$ dos nutrientes foram encontrados nas folhas da serapilheira produzida, sendo que, a concentração de nutrientes nas frações estudadas seguiu a ordem: folhas > galhos grossos > galhos finos > miscelânea.

Nesse caso, o maior teor é de N. Isso se deve ao fato deste elemento participar da maioria das reações de síntese de compostos (aminoácidos, proteínas, vitaminas, etc.), as quais ocorrem principalmente nas folhas devido à atividade fotossintética. Outro elemento que apresentou maior concentração nas folhas em relação às demais frações foi o K. Isto está relacionado ao fato deste elemento apresentar uma rápida ciclagem na relação solo-planta comparado aos outros nutrientes, por se tratar de um cátion monovalente (CALDEIRA, 2008), além de ser lavado da copa das árvores até o piso florestal pela ação da precipitação pluviométrica (PEREZ-MARIN e MENEZES, 2008).

Tabela 2 - Concentrações de nutrientes na serapilheira produzida em povoamento de Eucalyptus dunnii, Alegrete, RS.

Table 2 - Nutrient concentrations in the litterfall in Eucalyptus dunnii stand, Alegrete, RS state.

\begin{tabular}{|c|c|c|c|c|c|}
\hline \multirow{2}{*}{ Fração } & $\mathrm{N}$ & $\mathrm{P}$ & $\mathrm{K}$ & $\mathrm{Ca}$ & $\mathrm{Mg}$ \\
\hline & \multicolumn{5}{|c|}{$\mathrm{g} \mathrm{kg}^{-1}$} \\
\hline Folhas & $8,99 a^{*}$ & $0,64 \mathrm{a}$ & $4,54 \mathrm{a}$ & $14,14 \mathrm{a}$ & $1,94 \mathrm{~b}$ \\
\hline Miscelânea & $4,53 \mathrm{~b}$ & $0,20 \mathrm{~b}$ & $1,60 \mathrm{c}$ & $4,59 \mathrm{c}$ & $2,36 \mathrm{a}$ \\
\hline Galhos finos & $3,49 \mathrm{~b}$ & $0,14 \mathrm{c}$ & $2,93 \mathrm{~b}$ & $9,52 \mathrm{~b}$ & $2,08 \mathrm{a}$ \\
\hline Galhos grossos & $2,28 \mathrm{c}$ & $0,12 \mathrm{c}$ & $2,46 \mathrm{~b}$ & $11,74 \mathrm{a}$ & $2,15 \mathrm{a}$ \\
\hline
\end{tabular}

*Médias seguidas pela mesma letra na coluna não diferem entre si pelo teste Tukey ao nível de 5\% de probabilidade de erro

As maiores concentrações de $\mathrm{Ca}$ nas folhas estão relacionadas à baixa mobilidade no tecido vegetal, pois são as folhas maduras e senescentes que compõem a maior parte da serapilheira produzida; além disso, esse elemento é abundante nos tecidos lignificados, portanto, também pode ser encontrado em altas concentrações nos galhos grossos (SCHUMACHER et al., 2004).

\section{Quantidades de macronutrientes}

$\mathrm{Na}$ Tabela 3 estão apresentadas as quantidades de nutrientes devolvidos ao solo através da serapilheira produzida, nesse caso, observou-se que a magnitude de aporte seguiu a ordem: $\mathrm{Ca}>\mathrm{N}>\mathrm{K}>\mathrm{Mg}>\mathrm{P}$. 
Tabela 3 - Quantidade de nutrientes aportados através da serapilheira em povoamento de Eucalyptus dunnii em Alegrete, RS.

Table 3 - Amount of nutrients contributed by litterfall in Eucalyptus dunnii stand in Alegrete, RS state.

\begin{tabular}{ccccccc}
\hline \multirow{2}{*}{ Fração } & $\mathrm{N}$ & $\mathrm{P}$ & $\mathrm{K}$ & $\mathrm{Ca}$ & $\mathrm{Mg}$ & $\mathrm{S}$ \\
\cline { 2 - 7 } & \multicolumn{7}{c}{$\mathrm{kg} \mathrm{ha}^{-1}$} \\
\hline Folhas & 45,84 & 3,29 & 22,33 & 74,95 & 10,83 & 4,08 \\
Miscelânea & 3,97 & 0,17 & 1,31 & 3,88 & 2,27 & 0,31 \\
Galhos finos & 4,71 & 0,21 & 4,46 & 15,84 & 3,24 & 0,48 \\
Galhos grossos & 5,10 & 0,25 & 5,90 & 14,70 & 4,83 & 0,55 \\
\hline Total & 59,62 & 3,93 & 33,99 & 109,36 & 21,17 & 5,41 \\
\hline
\end{tabular}

Devido à sua predominante biomassa, as folhas contribuem em maior escala para a ciclagem de nutrientes (VIERA, 2012), sendo que, no presente estudo, esta fração representou cerca de $70 \%$ do retorno de nutrientes, seguido pelos galhos grossos (13\%), galhos finos (12\%) e miscelânea (5\%). Esta mesma ordem de transferência foi observada por Ashagrie e Zech (2013), que estudaram Eucalyptus globulus, Corrêa et al. (2013), estudando Eucalyptus dunnii, e por Silva et al. (2015), que estudaram Eucalyptus camaldulensis.

Kolm e Poggiani (2003), estudando a ciclagem de nutrientes em plantação de Eucalyptus grandis, e Hernández et al. (2009) encontraram valores semelhantes, havendo uma inversão na ordem em relação ao $\mathrm{Mg}$ e ao $\mathrm{K}$ $(\mathrm{Ca}>\mathrm{N}>\mathrm{Mg}>\mathrm{K}>\mathrm{P})$. A dominância do $\mathrm{Ca}$, em todos os trabalhos mencionados, pode ser explicada pela sua baixa mobilidade no floema, característica essa que dificulta a sua retranslocação durante a senescência.

\section{Conclusões}

A serapilheira pode ser caracterizada como uma fonte de devolução de nutrientes ao solo, sendo que sua produção é sazonal, com os maiores valores coincidindo com os períodos de maior temperatura da região.

Com relação à variação da deposição de serapilheira, a fração folhas é a que tem a maior contribuição, apesentando 52,7\%, seguida dos galhos grossos $(23,7 \%)$, galhos finos $(13,9 \%)$ e miscelânea $(9,7 \%)$.

O material depositado contribuiu de forma significativa para a manutenção do clico biogeoquímico, por meio da elevada devolução de macronutrientes ao solo, os quais, neste estudo apresentaram a seguinte ordem de contribuição: $\mathrm{Ca}>\mathrm{N}>\mathrm{K}>\mathrm{Mg}>\mathrm{P}$.

\section{Referências}

ALVARES, C. A. et al. Köppen's climate classification map for Brazil. Meteorologische Zeitschrift. v. 22, n. 6, p. 711-728, 2014.

ASHAGRIE, Y.; ZECH, W. Litter production and nutrient cycling in two plantations and a Podocarpus falcatus dominated natural forest ecosystems in south-eastern highlands of Ethiopia. African Journal of Agricultural Research. v. 8, n. 38, p. 4810-4818, 2013.

BALIEIRO, F. C. et al. Dinâmica da serapilheira e transferência de nitrogênio ao solo, em plantios de Pseudosamanea guachapele e Eucalyptus grandis. Pesquisa Agropecuária Brasileira, v. 39, n. 6, p. 597-601, 2004.

CALDEIRA, M. V. W. Quantificação de serapilheira e de nutrientes em uma Floresta Ombrófila Densa. Ciências Agrárias, v. 29, n. 1, p. 53-68, 2008. 
CARVALHO, R. R. Biomassa e nutrientes em um povoamento de Eucalyptus urograndis estabelecido em solo sujeito a arenização no sul do Brasil. 2014. 80 f. Dissertação (Mestrado em Engenharia Florestal) - Universidade Federal de Santa Maria, Santa Maria. 2014.

CIZUNGU, L. et al. Litterfall and leaf litter decomposition in a central African tropical mountain forest and Eucalyptus plantation. Forest Ecology and Management, v. 326, p. 109-116, 2014.

CQFS - RS/SC - Comissão de Química e Fertilidade do Solo. Manual de adubação e de calagem para os Estados do Rio Grande do Sul e de Santa Catarina. 11. ed. Sociedade Brasileira de Ciência do Solo - Núcleo Regional Sul. 376 p. 2016.

CORRÊA, R. S.; SCHUMACHER, M. V.; MOMOLLI, D. R. Deposição de serapilheira e macronutrientes em povoamento de Eucalyptus dunnii Maiden sobre pastagem natural degradada no bioma Pampa. Scientia Forestalis, v. 41, n. 97, p. 65-74, 2013.

EMBRAPA - Empresa Brasileira de Pesquisa Agropecuária. Sistema Brasileiro de Classificação de Solos. Embrapa Solos, $3^{\mathrm{a}} \mathrm{Ed}$. 342p., 2013.

FIGUEIREDO FILHO, A. et al. Avaliação Estacional da Produção de Serapilheira em uma Floresta Ombrófila Mista Localizada no Sul do Paraná. Ciência Florestal, v. 13, n. 1, p. 11-18, 2003.

HERNÁNDEZ, J. et al. Nutrient export and harvest residue decomposition, patterns of a Eucalyptus dunnii Maiden plantation in temperate climate of Uruguay. Forest Ecology and Management, v. 258, n. 2 p. 92- 99, 2009.

INKOTTE J. et al. Deposição de serapilheira em reflorestamentos de eucalipto e florestas nativas nas regiões Planalto e Oeste do Estado de Santa Catarina. Scientia Forestalis, v. 43, n.
106, p. 261-270, 2015.

KOLM, L.; POGGIANI, F. Ciclagem de nutrientes em povoamentos de Eucalyptus grandis submetidos à prática de desbastes progressivos. Scientia Forestalis, n. 63, p. 7993, 2003.

MARCHIORI, J. N. C. Fitogeografia do Rio Grande do Sul: enfoque histórico e sistemas de classificação. EST, 2002. 118p.

MIYAZAWA, M. et al. Análises químicas de tecido vegetal. In: SILVA, F. C. (Org). Manual de análises químicas de solo, plantas e fertilizantes. Embrapa Comunicação para Transferência de Tecnologia, 1999. Cap. 4, pag. 171-224.

MOREIRA, J. M. M. Á. P. et al. Importância e desempenho das florestas plantadas no contexto do agronegócio brasileiro. Floresta, v. 47, n. 1, p. 85-94, 2017.

PEREZ-MARIN, A. M.; MENEZES, R. S. C. Ciclagem de nutrientes via precipitação pluvial total, interna e escoamento pelo tronco em sistema agroflorestal com Gliricidia sepium. Revista Brasileira de Ciência do Solo, v. 32, p. 2573-2579, 2008.

SCHUMACHER, M. V. et al. Produção de serapilheira em uma floresta de Araucaria angustifolia (Bertol.) Kuntze no município de Pinhal Grande-RS. Revista Árvore, v. 28, n. 1, p. 29-37, 2004.

SILVA, J. C. M. Ciclagem biogeoquímica de nutrientes em Eucalyptus dunnii Maiden em uma microbacia hidrográfica experimental do bioma Pampa. 2014. 111 f. Tese (Doutorado em Recursos Florestais e Engenharia Florestal) Universidade Federal de Santa Maria, Santa Maria, 2014.

SILVA, C. F. et al. Deposition and nutritional quality of the litter of pure stands of Eucalyptus camaldulensis and Acacia mangium. 
Bioscience Journal, v. 31, n. 4, p. 1081-1091, 2015.

SILVA, F. A. S.; AZEVEDO, C. A. V. Versão do programa computacional ASSISTAT para o sistema operacional Windows. Revista Brasileira de Produtos Agroindustriais, v. 4, n. 1, p. 71-78, 2002.

TANG, G. et al. Accelerated nutrient cycling via leaf litter, and not root interaction, increases growth of Eucalyptus in mixed-species plantations with Leucaena. Forest Ecology Management, v. 310, n. 2, p. 45-53, 2013.

TEDESCO, M. J. et al. Análise de solos, plantas e outros materiais. Departamento de solos, UFRGS, Boletim Técnico, 1995. 118p.

TOLEDO, L. O.; PEREIRA, M. G. Dinâmica da deposição de serapilheira em florestas secundárias do município de Pinheiral, RJ. Floresta e Ambiente, v. 11, n. 1, p. 39-46, 2004.

VIERA, M.; SCHUMACHER, M. V. Deposição de serapilheira e de macronutrientes em um povoamento de acácia-negra (Acacia mearnsii De Wild) no Rio Grande do Sul. Ciência florestal, v. 20, n. 2, p. 225-233, 2010a.

VIERA, M.; SCHUMACHER, M. V. Teores e aporte de nutrientes na serapilheira de Pinus taeda L., e sua relação com a temperatura do ar e pluviosidade. Revista Árvore, v. 34, n. 1, p. 85-94, 2010b.

VIERA M. Dinâmica nutricional em um povoamento híbrido de Eucalyptus urophylla $x$ Eucalyptus globulus em Eldorado do Sul - RS, Brasil. 2012. 119 f. Tese (Doutorado em Engenharia Florestal) - Universidade Federal de Santa Maria, Santa Maria. 2012.

VIERA, M. et al. Deposição de serapilheira e nutrientes em plantio de Eucalyptus urophylla $\times$ E. globulus. Floresta e Ambiente, v. 21, n. 3, p. 327-338, 2014. 